\title{
Pitfall in the diagnosis of Lynch syndrome
}

\author{
Noriomi Matsumura ${ }^{1}$
}

Published online: 6 January 2021

(c) The Japan Society of Clinical Oncology 2021
This issue contains two case reports on the diagnosis and treatment of tumors that were apparently related to Lynch syndrome.

Toyota et al. reported a quadruple cancer caused by Lynch syndrome [1]. The patient had a gastric, ascending colon, sigmoid colon, and recto-sigmoid carcinoma. On immunostaining, all four cancers lacked MLH1/PMS2 and were thought to be Lynch's syndrome caused by a germline mutation of $M L H 1$. Such cancers are usually tested positive for MSI due to abnormal DNA mismatch repair. However, Toyota et al. performed MSI tests on all four of these cancers and interestingly, three of the cancers were MSI-high, but the gastric cancer was MSS. This suggests that it is important to use not only the MSI test but also immunostaining to screen for Lynch syndrome. In addition, in Japan, pembrolizumab is currently approved for MSI-high tumors diagnosed by PCR or next-generation sequencing, but not for MMR deficiency by immunostaining. And the accuracy of PCR-based MSI testing is not $100 \%$. Therefore, if a MSI test by PCR performed for the companion diagnosis of pembrolizumab is negative and there is an MMR deficiency on immunostaining, a MSI test by next-generation sequencing should be performed.

Yoshihama et al. reported a patient with a family and medical history of seemingly Lynch syndrome who underwent germline multigene panel testing and was a carrier of the $B R C A 2$ pathogenic variant, not the pathogenic variant of the mismatch repair gene [2]. Importantly, they stated that conventional genetic risk assessment could not determine that the risk of HBOC was high. Their report indicates that a more comprehensive search by multigene panel testing, not just for mismatch repair genes, is important, even if Lynch syndrome is considered.

Molecular testing in cancer is advancing rapidly, and efficient use of these tests will greatly advance cancer care. It is expected that a variety of new molecular tests will be approved for insurance and commonly used in the future.

\section{References}

1. Toyota S, Nakanishi R, Miyashita Y et al (2020) Quadruple gastrointestinal cancer with discordance of mismatch repair protein deficiency and microsatellite instability suggesting Lynch syndrome. Int Cancer Conf J. https://doi.org/10.1007/s13691-02000457-9

2. Yoshihama T, Hirasawa A, Sugano K et al (2020) Germline multigene panel testing revealed a BRCA2 pathogenic variant in a patient with suspected Lynch syndrome. Int Cancer Conf J. https ://doi.org/10.1007/s13691-020-00449-9

Publisher's Note Springer Nature remains neutral with regard to jurisdictional claims in published maps and institutional affiliations.
Noriomi Matsumura noriomi@med.kindai.ac.jp

1 Faculty of Medicine, Kindai University, Osakasayama, Japan 